\title{
Researching the changing social pattern of cardiovascular diseases
}

In many Western countries, despite declining overall mortality from cardiovascular diseases (CVDs) since the 1970 s, the relative difference in disease rates between the richest and poorest groups has widened. ${ }^{1}$ Both stroke and coronary heart disease (CHD), the main contributors to CVD mortality, have a multifactorial aetiology. However, debate continues about the relative importance of a large number of established and putative risk factors. ${ }^{12}$ Reducing health inequalities has become an increasingly important policy objective over the past 20 years $^{34}$ and interest has focused on how observed social inequalities in CVD can be reduced. ${ }^{4}$

The study by Bartley et $a l^{6}$ presents an ecological analysis that aims to shed some light on this question. Using data on men aged 20-64 years from two unrelated cross sectional studies 10 years apart they have compared the prevalence and social distribution of a range of behavioural, physiological and other risk factors. To examine the degree of inequality in the distribution of risk factors they used the Erikson-Goldthorpe (E-G) social class schema ${ }^{7}$ and calculated the relative index of inequality (RII) ${ }^{8}$ across social categories at each time period for each risk factor. These are relatively new methods that offer some methodological advantages. ${ }^{6}$

However, such studies are not without limitations. For example, this study focuses only on men because the measurement and interpretation of social categories among women remains problematic. ${ }^{9}$ The study is also limited to those aged 20-64 years, which makes it difficult to interpret the findings in relation to mortality at all ages; the temporal relation between risk factors and disease onset is still poorly understood. ${ }^{26}$ In addition, the two surveys used different methods, including different questions and risk factor definitions. The ecological nature of the analysis also means we should be particularly cautious in its interpretation; the two surveys included different people and no direct relation between risk and mortality is explored.

Social inequalities in most cardiovascular risk factors have been reported throughout the world ${ }^{10}$ and this study confirmed such variations with respect to all behavioural, most physiological and important predisposing morbidity (angina, diabetes) factors measured. ${ }^{6}$ However, no systematic evidence of widening inequality (as measured by RII) in risk factors between the two time periods was found. Only diastolic blood pressure showed a significant change in RII, and this was a narrowing of a relatively small inequality between 1984 and 1993.

The authors conclude that healthier lifestyles have not been adopted at a faster rate by higher social classes over this period. Despite this, the observed widening mortality gap remains. ${ }^{6}$ Two explanations are proposed for this lack of observed relation between risk behaviours and mortality. Firstly, there may be a lag time effect - recent behaviour changes will take many years to affect mortality. Alternatively, the authors suggest that healthier behaviours confer greater benefit (greater reduction in CVD mortality) on those in higher social classes. ${ }^{6}$

Neither of these are entirely satisfactory explanations. Both suggest that health behaviours are the most important determinants of CVD mortality. Yet in analyses of CHD risk, for example, typically only around half of the variance in mortality has been attributed to known risk factors, of which health related behaviours form only a part. ${ }^{12}$ It is possible, therefore, that social inequalities in CVDs may be explained better by other, yet to be determined, factors. Equally, it is important to recognise that any longitudinal changes in socioeconomic variation in CVD mortality may be unrelated to health behaviours or other established risk factors.

One set of factors that we do know about and that are known often to be patterned socioeconomically are those affecting access to and uptake of health services for the clinical management of CVDs. ${ }^{11}{ }^{12}$ Clearly improvements in management and prevention of CVDs, including thrombolysis for heart attacks, routine computed tomography of stroke victims, screening techniques and the use of clinical guidelines, may have made an impact on overall mortality; and there is evidence to suggest that this impact has been greater for higher social groups. ${ }^{512}$

If this is an important part of the explanation for the observed widening of the mortality gap then, in the absence of worsening or persisting social inequalities in behavioural risk factors, effort should be invested in reducing social inequalities in the effective clinical management of CVDs. This will require more applied research and development focusing on structural issues in service delivery and professional behaviour change.

Meanwhile, further research is needed to develop a clearer understanding of the relation between socioeconomic inequalities and biological outcomes, such as wide disparities in CVD mortality. What we learn from studies such as that by Bartley et al is that our knowledge is incomplete and certainly insufficient to make important policy decisions concerning the appropriate targeting of resources on treatment and prevention. Relevant knowledge can only come from prospective studies that measure and compare risk, incidence and mortality in different social groups and have the capacity to explore novel aetiological hypotheses. Such studies need to be underpinned by appropriate conceptual models for the relations between factors thought to influence mortality from CVDs. Hierarchical conceptual frameworks provide a useful tool for this purpose. ${ }^{13}$ Such frameworks enable us to describe possible causal pathways in a model, including both proximal determinants (immediately and usually independently causal) and distal determinants (usually indirectly causal and mediated through other factors) in appropriate hierarchies. ${ }^{13}$ In the case of CHD or stroke for example, such a framework would include not only risk behaviours and physiological factors, but also educational, policy or health technology interventions for primary and secondary prevention, screening, acute and chronic disease management, access to facilities and services, and social and demographic factors. In such frameworks the size and direction of relations between factors leading causally to variations in disease incidence can be separated from those responsible for differing responses to efforts to prevent the disease and those affecting disease outcome and case fatality, thus enabling us to get closer to understanding the complex web of factors leading to differential mortality in populations. Conceptual frameworks can also be tested empirically, ${ }^{13}{ }^{14}$ but this will require major investment in research and, in particular, large scale studies of representative populations over long time periods.

MARTIN WHITE

Health Promotion Research Group, Department of Epidemiology and Public Health, School of Health Sciences, The Medical School, University of Newcastle Upon Tyne NE2 4HH (martin.white@ncl.ac.uk) 
1 Kaplan G, Keil J. Socio-economic indicators and mortality from coronary heart disease: a review of the literature. Circulation 1992;88:1973-98.

2 Kannel W. An overview of the risk factors for cardiovascular disease. In: Buck C, Llopis A, Najera E, et al, ed. The challenge of epidemiology: issues and selected readings. Washington: Pan American Health Organisation, 1988 699-718.

3 World Health Organization. Global Strategy for Health For All by the year 2000. Geneva: World Health Organization, 1980.

4 Department of Health. Saving Lives: Our Healthier Nation. London: The Stationery Office, 1999.

5 Acheson D. Independent Inquiry into inequalities in Health. London: HMSO, 1998.

6 Bartley M, Fitzpatrick R, Firth D, et al. Social distribution of cardiovascular disease among men in England 1984-1993. F Epidemiol Community Health 2000;54:806-14.

7 Erikson R, Goldthorpe J. The constant flux. Oxford: Clarendon, 1992.

8 Kunst A, Mackenbach J. Measuring socio-economic inequalities in health. Copenhagen: World Health Organization, 1994.
9 Bartley M, Sacker A, Firsth D, et al. Social positions, social roles and women's health in England: changing relationships 1984-1993. Soc Sci Med en's health in Englar

10 Nogueira A, Marcopito L, Lanas F, et al. Socio-economic status and risk factors for cardiovascular disease - a multi-centre collaborative study in the International Clinical Epidemiology Network (INCLEN). $\mathcal{F}$ Clin Epidemiol 1994;47:1401-9.

11 Brown A, Garber A. A concise review of the cost effectiveness of coronary heart disease prevention. Med Clin North Am 2000;84:279-97.

12 Capewell S, Morrison C, McMurray J. Contribution of modern cardiovascular treatment and risk factor changes to the decline of coronary heart disease in Scotland between 1975 and 1994. Heart 1999;81:280-6.

13 Victoria C, Huttly S, Fuchs S, et al. The role of conceptual frameworks in epidemiological analysis: a hierarchical approach. Int $\mathcal{f}$ Epidemiol 1997;26: $224-7$.

14 Lamont D, Parker L, White $\mathrm{M}$, et al. Risk of cardiovascular disease measured by carotid intima-media thickness at age 49-51: lifecourse study. BM7 2000;320:273-8. 